\section{STRUCTURAL STUDIES OF ANTIBODIES IMPLICATED IN} CHAGAS DISEASE

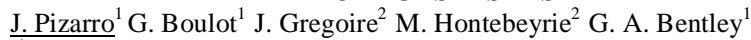
${ }^{1}$ Institut Pasteur Unite Dimmunologie Structurale 25 Rue Du Dr. Roux PARIS

75724 FRANCE ${ }^{2}$ Departement dImmunologie, Institut Pasteur, Paris, France region, is caused by the parasite Trypanosoma cruzi. A major consequence of infection is the occurrence of long-term symptoms of chronic cardiomyopathy. Antibodies induced by the ribosomal protein $\mathrm{P} 2 \beta$ from $T$. cruzi (TcP2 $\beta$ ) crossreact with a specific epitope on the human $\beta 1$-adrenergic receptor of cardiomyocytes, suggesting an autoimmune mechanism for this pathology. In order to characterise the phenomenon further, we are studying an anti-TcP2 $\beta$ monoclonal antibody (mAb), 17.2, and its interaction with both the cognate and cross-reactive antigens. The monoclonal antibody 17.2, which was raised against the recombinant ribosomal protein, recognises an epitope at the Cterminus of the molecule. In addition, mAb 17.2 simulates rat cardiomyocytes, and cross-reacts with a peptide carrying the $\beta 1$-adrenergic receptor epitope that is implicated in the cardiomyopathy of Chagas disease. We have determined the crystal structure of Fab 17.2 as a complex with a peptide containing the ribosomal epitope. Crystallisation trials of Fab 17.2 with TcP2 $\beta$ and a peptide containing the $\beta 1$-adrenergic receptor epitope are in progress to complement our initial structural results. COMPLEX
Chagas disease, which affects 12 million people living in the Latin American

Keywords: FAB STRUCTURE CHAGAS DISEASE PEPTIDE

Acta Cryst. (2002). A58 (Supplement), C282

STRUCTURE OF TWO NEW T CELL RECEPTORS HIGHLIGHTS THE RECOGNITION OF ALLOGENEIC MHC MOLECULES J.-B. Reiser ${ }^{1}$ C. Darnault ${ }^{1}$ C. Gregoire ${ }^{2}$ T. Mosser ${ }^{2}$ A. Guimezanes ${ }^{2}$ A.-M. Schmitt-Verhults $^{2}$ J.C. Fontecilla-Camps ${ }^{1}$ G. Mazza ${ }^{2}$ B. Malissen ${ }^{2}$ D. Housset ${ }^{1}$ ${ }^{1}$ Laboratoire de Cristallographie et Cristalogenese des Proteines, Institut de Biologie Structurale J.-P. Ebel, CEA-CNRS-UJF 41, Rue Jules Horowits GRENOBLE CEDEX 1 F-38027 FRANCE ${ }^{2}$ Centre dImmunologie de Marseille-Luminy, INSERM-CNRS-Univ.Med., Parc Scientifique de Luminy, Case 906, F-13288 Marseille Cedex 9, France

The $\mathrm{T}$ lymphocytes are the main actors of the cellular immune response. They protect the organism against pathogens internalized by cells and against some kinds of cancer. To achieve this goal, T cells display at their surface Ig-like receptors (TCRs) capable of recognizing peptide fragments derived from foreign proteins and presented at the infected cell surface by Major Histocompatibily Complex (MHC) coded proteins. Moreover, many TCRs selected to crossreact with several peptides bound to self-MHC molecules, also express reactivity towards intraspecies allelic variants of these MHC molecules. This property termed allo-reactivity is responsible for graft rejection and graft-versus-host diseases. With the aim of understanding the structural basis of the TCR specificity vis-a-vis the peptide and the MHC molecule, we have determined the crystal structures of 2 murine TCRs (BM3.3 and KB5-C20 at $2.5 \AA$ and $2.7 \AA$ resolution respectively) in complex with a self-octapeptide bound to the allogeneic $\mathrm{H}-2 \mathrm{~Kb}$ MHC class I molecule. These two new $\mathrm{TCR} /$ peptide/MHC complexes show that alloresponse requires self- and alloMHCs to have nearly identical TCR facing residues and that allorecognition is essentially mediated by the different self-peptide repertoire presented by the allo-MHC molecule. The large KB5-C20 CDR3 $\beta$ conformational change observed upon pMHC binding shows that a long CDR3 $\beta$ loop can interact with the peptide central residues without preventing the TCR to dock onto the pMHC in a canonical way. However, the remarkable diversity of peptide readout illustrated by the available ternary complex structures highlights the difficulty we shall encounter to decipher the structural basis of TCR crossreactivity.

\section{Keywords: T CELL RECEPTOR ALLORECOGNITION TCR/PMHC COMPLEX}

\section{Acta Cryst. (2002). A58 (Supplement), C282}

\section{HIGH RESOLUTION NATIVE AND LIGAND-BOUND STRUCTURES} OF AN ACTIVE TRIMERIC FRAGMENT OF HUMAN SP-D A.K. Shrive ${ }^{1}$ H.A. Tharia ${ }^{1}$ C. Martin $^{1}$ I. Burns ${ }^{1}$ P.J. Rizkallah ${ }^{2}$ U. Kishore ${ }^{3}$ P. Strong $^{3}$ K.B.M Reid ${ }^{3}$ T.J. Greenhough ${ }^{1}$

${ }^{1}$ Keele University School of Life Sciences Huxley Building KEELE STAFFORDSHIRE ST5 5BG UK ${ }^{2}$ CLRC Daresbury Laboratory, Daresbury, Warrington, Cheshire WA4 4AD, UK ${ }^{3} \mathrm{MRC}$ Immunochemistry Unit, Department of Biochemistry, Oxford University, Oxford OX1 3QU, UK

The collectins are a group of C-type lectins involved in a variety of defence functions including defence against invading microorganisms, immune system regulation and recognition of cell surface receptors on immune effector cells. The human collectins bind in a fairly non-specific Ca-dependent manner to carbohydrate structures on a wide range of pathogens. The recombinant human SP-D fragment rhSP-D, a homotrimer whose recognition, effector and antimicrobial properties have significant medical potential, comprises a small portion of the collagen-like domain, the $\alpha$-helical coiled coil neck region and three carbohydrate recognition domains. rhSP-D, which acts as a therapeutic agent in mouse models, appears to have significant biological activity and has been shown to interact with recombinant fragments of its putative cell surface receptor. We present here the high resolution crystal structures of ligand-bound rhSP-D to $1.4 \AA$, the first structural description of ligand-binding to the trimeric assembly of a collectin, and native rhSP-D to $1.6 \AA$. The ligand extends from the CRD with the terminal glucose glc1 bound to calcium and to the protein through $\mathrm{O} 3$ and O4. Relatively short van der Waals interactions between glc1 and $\operatorname{Arg} 343$ appear to contribute to ligand recognition and binding. The results suggest monosaccharide recognition and confirm the asymmetric positioning of Tyr228 although there are significant differences between the two structures associated with the interactions of this residue and with associated interactions between the neck region and the CRD. These differences include a putative calcium ion, not present in the ligand-bound structure, located on the trimeric axis.

Keywords: INNATE IMMUNITY, C-TYPE LECTIN, THERAPEUTIC AGENT

\section{Acta Cryst. (2002). A58 (Supplement), C282}

STRUCTURAL, KINETIC AND BIOCHEMICAL CHARACTERIZATION OF THE OXIDATION STATES OF HUMAN PROTEIN TYROSINE PHOSPHATASE 1B: IMPLICATIONS FOR REDOX REGULATION OF PHOSPHATASE ACTIVITY

C. Abad-Zapatero ${ }^{1}$ T. Lubben ${ }^{3}$ J. Severin $^{2}$ E. Hebert ${ }^{2}$ T. Ahmed ${ }^{4}$ R. Johnson ${ }^{4}$

K. Stewart ${ }^{5}$ C. Hutchins ${ }^{5}$ T. Oie ${ }^{5}$ R. Smith ${ }^{2}$

${ }^{1}$ Abbott Laboratories, Protein Crystallography D-R46y, Ap-10, L-07

Department of Structural Biology, D-R46Y, AP-10, L-07 100 Abbott Park Rd. ABBOTT PARK IL 60064-6098 USA ${ }^{2}$ Protein Biochemistry ${ }^{3}$ Diabetes

Research ${ }^{4}$ Analytical Chemistry ${ }^{5}$ Molecular Modeling

Sensitivity of several enzyme classes to the redox environment of the cell or to oxidative stress suggests that redox regulation of enzyme activity may play an important role in cellular processes. Evidence is emerging for the modulation of PPases by controlled redox processes (Rusnak \& Reiter, TIBS 2000, 25:527529). In particular, PTPases have been shown to be extremely sensitive to minute concentrations of $\mathrm{H}_{2} \mathrm{O}_{2}$ due to the oxidation of their active site residue Cys215 within the tyrosine phosphatase fingerprint sequence HCXXXR (Denu \& Tanner, Biochemistry, 1998, 37:5633-5642). It has been reported that insulin-stimulated hydrogen peroxide inhibits hPTPase1B in vivo and enhances early events in the insulin cascade (Zilbering et al. J. Biol. Chem. 2001, 276: 21938-21942). A progressive oxidation of Cys215 through the sulfenic ($\mathrm{SOH})$, sulfinic (-SOOH) and sulfonic (-SOOOH) states has been suggested as the normal oxidative pathway of PTPases with the corresponding inactivation. A reversal of the sulfenic state via glutathione (Denu \& Tanner, Biochemistry, 1998, 37:5633-5642; Barret et al. Biochemistry, 1999, 38:6699-6705) might be a possible regulatory mechanism in the cell. Structural characterization of these transient states has been lacking. A critical issue has been how the sulfenic state is stabilized and/or prevented from further oxidation (Claiborne et al. FASEB J., 1993, 7: 1483-1490).

We present here the structural characterization of the three oxidation states of the active site residue Cys 215 in hPTPase1B achieved by controlled oxidation of crystals of the native enzyme. The implications for the redox regulation of phosphatase activity will be presented. 